\title{
Descripción de los esquemas de inicio de la alimentación enteral en el recién nacido pretérmino en un hospital privado de tercer nivel
}

\section{Description of the enteral feeding initiation schemes in preterm infants in a Private tertiary Hospital}

\author{
Gabriela Tijerina Tijerina, ${ }^{*}$ Elías Duck Hernández, ${ }^{\ddagger}$ David Oldak Skvirsky, ${ }^{\S}$ \\ Lucía Escobedo Berumen, "Sergio Ramírez Nova*
}

Citar como: Tijerina TG, Duck HE, Oldak SD, Escobedo BL, Ramírez NS. Descripción de los esquemas de inicio de la alimentación enteral en el recién nacido pretérmino en un hospital privado de tercer nivel. An Med (Mex). $2021 ; 66$ (1): 13-18. https://dx.doi. org/10.35366/99484

\section{RESUMEN}

Introducción: El manejo nutricional en el recién nacido pretérmino es crucial para la salud y bienestar a largo plazo. Existen muchas razones del fracaso del crecimiento postnatal y no se ha demostrado si existe algún protocolo de alimentación óptimo. Objetivo: Describir el tiempo transcurrido en alcanzar la alimentación enteral completa en volumen y contenido calórico así como identificar cuál es el método de inicio de vía oral más efectivo para los recién nacidos pretérmino. Material y métodos: Estudio longitudinal retrospectivo-observacional descriptivo. Se incluyeron recién nacidos < 33.6 semanas de gestación de enero de 2019 a marzo de 2020 , se registró el volumen de incremento hasta llegar al requerimiento calórico total. Se indagaron características nutricionales y se agruparon

\section{ABSTRACT}

Introduction: Nutritional management in the preterm infant is crucial for long-term health and well-being. Although there are many reasons for postnatal growth failure, there is still no protocol that guarantees an optimal enteral feeding advancement. Objective: Describe the time to reach complete enteral feedings in volume and caloric content, as well as to identify the most effective feeding advancement. Material and methods: Retrospective, observational, descriptive longitudinal study. We included preterm infants $<33.6$ of gestational age, from January 2019 to March 2020, and recorded the feeding advancements from birth until they reached total caloric requirement. Nutritional characteristics were investigated and grouped into 3 types of feeding advancements: group 1: 5-20

\footnotetext{
* Médico residente de Neonatología del Hospital Ángeles Lomas, Estado de México.

‡ Pediatra Neonatólogo del Hospital Ángeles Lomas, Estado de México.

$\S$ Jefe de la División de Neonatología del Hospital Ángeles Lomas, Estado de México.

१ Médico residente de Gastroenterología Pediátrica en el Instituto Nacional de Pediatría, Ciudad de México.
}

Correspondencia: Gabriela Tijerina Tijerina

Lago Tangañica Núm. 59,

Col. Granada, 11520

Alcaldía Miguel Hidalgo, CDMX.

E-mail: gabriela.tijerinat@gmail.com
Recibido: 27/05/2020. Aceptado: 04/02/2021.

\section{Abreviaturas:}

DVEU = Días de vida extrauterina.

$\mathrm{ECN}=$ Enterocolitis necrosante.

$\mathrm{LM}=$ Leche materna.

NPT $=$ Nutrición parenteral.

$\mathrm{PCA}=$ Persistencia del conducto arterioso.

$\mathrm{PT}=$ Pretérmino.

$\mathrm{RN}=$ Recién nacido.

$\mathrm{RPM}=$ Ruptura prolongada de membranas . 
según los incrementos de la vía oral: grupo 1: $5-20 \mathrm{~mL} / \mathrm{kg} /$ día, grupo 2: 20-30 mL/kg/día, y grupo $3:>30 \mathrm{~mL} / \mathrm{kg} /$ día Resultados: 37 recién nacidos con promedio de 31.2 semanas de gestación. El grupo 1 con avances lentos tardó $18.4 \pm 6.66$ días en llegar a los requerimientos calóricos totales, el grupo 2 tardó $10.3 \pm 8.19$ días, y el grupo 3 con avances más rápidos tardó $7.89 \pm 3.65$. Grupo 1 requirió nutrición parenteral por $13.4 \pm 7.25$ y el grupo 3 por $3.2 \pm 3.7$ días; 14 pacientes que no requirieron nutrición parenteral. No encontramos asociación entre enterocolitis necrosante y aumentos rápidos. Conclusiones: Los incrementos rápidos en la vía enteral de (> $30 \mathrm{~mL} / \mathrm{kg} /$ día) reducen el tiempo en llegar a los requerimientos calóricos totales y el uso de nutrición parenteral. Esta práctica está asociada a menos comorbilidades.

Palabras clave: Pretérmino, nutrición neonatal, terapia intensiva neonatal.

Nivel de evidencia: III

\section{INTRODUCCIÓN}

La nutrición del recién nacido $(\mathrm{RN})$ pretérmino $(\mathrm{PT})$ tiene como objetivo promover un crecimiento similar al que ocurre de manera intrauterina sin imponer estrés en las funciones metabólicas y endocrinas inmaduras. ${ }^{1}$ El manejo nutricional es crucial para su salud y bienestar a largo plazo y la nutrición enteral siempre será la preferida y de elección en los primeros días de vida, ya que promueve la adaptación endocrina, maduración en la motilidad intestinal y el funcionamiento inmunológico, por ello la importancia de evitar retrasar su inicio. ${ }^{2}$ En la práctica, esto es difícil de lograr por la inmadurez de los sistemas antes mencionados, resultando en un retraso en el inicio de la alimentación enteral y su consecuente retraso en el crecimiento extrauterino. Esta nutrición subóptima en un periodo tan importante puede tener consecuencias en el desarrollo neurológico y programación metabólica. ${ }^{2}$

El desarrollo de la nutrición parenteral (NPT) y el temor a la enterocolitis necrosante (ECN) desencadenó como «rutina» el inicio tardío de la alimentación enteral en los prematuros de alto riesgo en la década del 80. En los últimos 15 años ha cambiado significativamente la edad de inicio del aporte enteral en el prematuro de muy bajo peso al nacimiento. Los estudios sobre los efectos de los nutrientes y el ayuno prolongado en la fisiología gastrointestinal han cuestionado esta práctica clínica y en la última década se han realizado estudios controlados que fundamentan el uso precoz del aporte enteral. ${ }^{3}$ Este periodo de deficiencia nutricional se considera como ineludible y puede llevar a una desnutrición tempra-
$\mathrm{mL} / \mathrm{kg} /$ day; group 2: 20-30 $\mathrm{mL} / \mathrm{kg} /$ day; and group 3: $>30$ $\mathrm{mL} / \mathrm{kg} /$ day. Results: 37 newborns with an average of 31.2 of gestational age. $75.7 \%$ with enteral stimulation the first 72 hours of life. Group 1 with slow feeding advancements took $18.4 \pm 6.66$ days to reach total caloric requirements. Group 2 took an average of $10.3 \pm 8.19$ days. Group 3 with rapid advancements took $7.89 \pm 3.65$. Group 1 required parenteral nutrition for almost two weeks (13.4 \pm 7.25 days) and group 3 less than one week (3.2 \pm 3.7 days); 14 patients did not require parental nutrition. Conclusions: Rapid feeding advancements (> $30 \mathrm{~mL} / \mathrm{kg} /$ day) reduce the time to reach the total caloric requirements and the use of parental nutrition. This practice does not increase the risk of necrotizing enterocolitis. On the contrary, it is associated with less comorbidities.

Keywords: Preterm birth, neonatal nutrition, neonatal intensive care.

Level of evidence: III

na que prolonga la estancia hospitalaria y propicia el riesgo de infección. Un metaanálisis de Cochrane concluye que retrasar la alimentación enteral no se asocia con reducción de ECN o mortalidad y por el contrario, retrasa la meta de peso y de lograr el aporte enteral calórico completo del recién nacido, ${ }^{4,5}$ lo que resulta en que estos pacientes se egresen de la terapia intensiva neonatal con peso en percentil < 10 para su edad, incluso cuando se hace el mejor esfuerzo por su manejo nutricional, ${ }^{2}$ y retrasando de 14 a 17 días para recuperar su peso al nacimiento, haciéndolos más susceptibles a déficits nutricionales postnatales. $^{4}$

La recomendación es iniciar la alimentación enteral lo más pronto posible, el número de días y el volumen para iniciarla y la velocidad con la cual se debe incrementar continúa en discusión. ${ }^{4,6} \mathrm{El}$ volumen de inicio de la alimentación enteral se ha recomendado entre 12 y $20 \mathrm{~mL} / \mathrm{kg} /$ día, con aumentos de volumen de 10 a $35 \mathrm{~mL} / \mathrm{kg} /$ día. Existen esquemas de inicio reportados que varían desde las primeras 24-72 horas o hasta cinco días. El momento de inicio parece depender principalmente de la inmadurez y patologías presentes durante los primeros días de vida así como el riesgo de posibles complicaciones. ${ }^{3}$ Sin embargo, los incrementos lentos se asocian hasta en $72 \%$ con el uso prolongado de NPT y sus complicaciones (sepsis, colestasis y enfermedad ósea metabólica del prematuro), ${ }^{6,7}$ mismas que con el inicio de la vía oral, la función hepática, la enfermedad metabólica ósea, los días de estancia hospitalaria, y la ganancia de peso mejoran. ${ }^{8}$

Los requerimientos calóricos totales de un $\mathrm{RN}$ $\mathrm{PT}$ son de aproximadamente $120 \mathrm{Kcal} / \mathrm{kg} /$ día que 
corresponden aproximadamente a $180 \mathrm{~mL} / \mathrm{kg} /$ día de leche materna (LM), con un aporte proteico correspondiente de $3-3.8 \mathrm{~g} / \mathrm{kg} /$ día. ${ }^{9}$ Los estudios que se han hecho aún no han podido definir un esquema óptimo. Se discute aún si es mejor hacer incrementos rápidos (30-40 mL/kg/día) o lentos (15-20 mL/kg/día), el tipo de leche, el intervalo entre tomas, entro otros. ${ }^{10,11}$

En nuestra unidad de cuidados intensivos el médico neonatólogo encargado del paciente decide de acuerdo con su «experiencia» cómo y cuándo iniciar la vía oral en el prematuro, por lo que son diferentes esquemas los que se utilizan.

El objetivo de este estudio es describir el tiempo transcurrido en llegar a la alimentación enteral completa en volumen y requerimiento calórico desde el nacimiento. Como objetivo secundario identificar cuál es el método de inicio de vía oral más efectivo para llegar en menor tiempo a su requerimiento calórico completo y observar factores que retrasen el incremento de la vía oral y/o el avance de éste así como las comorbilidades asociadas.

\section{MATERIAL Y MÉTODOS}

Este estudio longitudinal retrospectivo-observacional descriptivo se realizó en una terapia intensiva neonatal privada en el Estado de México durante el periodo del 01 de enero de 2019 al 31 de marzo de 2020.

Se incluyeron todos los RN PT $<33.6$ semanas de gestación nacidos en el hospital durante el periodo establecido. Se excluyeron pacientes con patología quirúrgica abdominal, malformaciones orales, asfixia perinatal, ventilación con alta frecuencia y se eliminaron los pacientes que fallecieron antes de llegar a completar la vía enteral, que se trasladaron a otra institución antes de llegar a sus requerimientos calóricos completos o pacientes con expediente incompleto.

En un inicio recibieron estímulo enteral y posteriormente se agruparon en tres esquemas según los incrementos de la vía oral: grupo 1: $5-20 \mathrm{~mL} / \mathrm{kg} /$ día, grupo 2: 20-30 mL/kg/día, y grupo 3: > $30 \mathrm{~mL} / \mathrm{kg} /$ día y de acuerdo al peso de nacimiento $(<1,000 \mathrm{~g}, 1,000$ $1,500 \mathrm{~g} \mathrm{y}>1,500 \mathrm{~g})$. Se analizaron las características nutricionales del paciente hasta llegar al requerimiento calórico meta. ( $120 \mathrm{Kcal} / \mathrm{kg} /$ día).

El proyecto fue aprobado por el comité de ética en investigación del hospital con el número 338/2020.

\section{RESULTADOS}

Se incluyeron 37 pacientes con una edad gestacional promedio de 31.2 semanas de gestación (27.5-33 se- manas de gestación). Las características de los $\mathrm{RN}$ se describen en la Tabla 1. No hubo predominio de sexo, 19 masculinos y 18 femeninos, $5.4 \%$ de los $\mathrm{RN}$ tuvieron peso $<1,000 \mathrm{~g}, 48.6 \%$ pesaron entre $1,000-1,500$ g y $45.9 \%$ con peso $>1,500$ g. De las comorbilidades encontradas, $27 \%$ tuvieron ruptura prolongada de membranas, $10 \%$ diabetes gestacional, hipotiroidismo, colestasis y preeclampsia (5.4\% respectivamente), sólo un paciente con antecedente de corioamnioitis y uno con síndrome de Hellp. De los pacientes, $45.9 \%$ no tienen antecedentes de comorbilidad materna.

En cuanto a las características nutricionales: $75.7 \%$ de los RNPT iniciaron estímulo enteral durante las primeras 72 horas de vida. Los pacientes se alimentaron en bolo y con una frecuencia de cada tres horas en $70.3 \%$ de los casos y el resto cada cuatro horas (29.7\%) (Tabla 2). El uso de NPT fue de 7.5 \pm 8.2 días; sin embargo, hubo 14 pacientes que no la requirieron y éstos tenían peso $>1,000 \mathrm{~g}$. Los días que tardaron en llegar a los requerimientos calóricos totales fueron $12.32 \pm 8.1$ días.

La mayoría recibió alimentación combinada, es decir, se ofrecía LM y alguna fórmula de las previamente mencionadas y esto sucedió en $67.5 \%$ de los casos, $8.1 \%$ recibió leche materna exclusiva y $24.3 \%$ recibió fórmula exclusiva. Dentro de las fórmulas utilizadas, $67.6 \%$ de los pacientes se alimentaron con fórmula hidrolizada, $10.8 \%$ con fórmula para prematuro; $10.8 \%$ con fórmula de inicio y $2.7 \%$ con fórmula elemental.

En la Tabla 3 se agruparon en tres grupos de acuerdo con el esquema de incremento que se utilizó,

Tabla 1: Características demográficas.

\begin{tabular}{lc}
\hline & $\mathrm{n}(\%)$ \\
\hline Sexo & \\
Masculino & $19(51.4)$ \\
Femenino & $18(48.6)$ \\
Semanas de gestación & $31.2 \pm 1.6$ \\
Peso al nacer (g) & \\
$<1,000$ & $2(5.4)$ \\
$1,000-1,500$ & $18(48.6)$ \\
$>1,500$ & $17(45.9)$ \\
Comorbilidad materna & \\
Hipotiroidismo & $2(5.4)$ \\
Diabetes & $4(10.8)$ \\
Colestasis & $2(5.4)$ \\
Corioamnioitis & $1(2.7)$ \\
Preeclampsia & $2(5.4)$ \\
Síndrome de Hellp & $1(2.7)$ \\
Ruptura prolongada de membranas & $10(27)$ \\
Sin comorbilidad & $17(45.9)$ \\
\hline
\end{tabular}


Tabla 2: Características nutricionales.

\begin{tabular}{lc}
\hline & $\mathrm{n}(\%)$ \\
\hline Inicio de vía oral (horas) & \\
$0-72$ & $28(75.7)$ \\
$>72$ & $9(24.3)$ \\
Frecuencia (horas) & \\
3 & $26(70.3)$ \\
4 & $11(29.7)$ \\
Días de uso de nutrición parenteral & $7.51 \pm 8.2$ \\
Días de vida extrauterina para llegar al & $12.32 \pm 8.10$ \\
requerimiento calórico total & \\
Tipo de alimentación & \\
Fórmula exclusiva & $9(24.3)$ \\
Leche materna exclusiva & $3(8.1)$ \\
Alimentación mixta & $25(67.5)$ \\
Tipo de fórmula & \\
$\quad$ Fórmula para prematuro & $4(10.8)$ \\
Fórmula de inicio & $4(10.8)$ \\
Fórmula hidrolizada & $25(67.6)$ \\
Fórmula elemental & $1(2.7)$ \\
\hline
\end{tabular}

en el grupo 1 incrementos de 5 a $20 \mathrm{~mL} / \mathrm{kg} /$ día con un total de 11 pacientes, en el grupo 2,17 pacientes con incrementos de 20 a $30 \mathrm{~mL} / \mathrm{kg} /$ día y por último nueve pacientes en el grupo 3 con los incrementos de 30 a $50 \mathrm{~mL} / \mathrm{kg} /$ día. No hubo diferencias en la edad gestacional de acuerdo con cada grupo, en su mayoría la media fue de 30 a 31 semanas de gestación. Se observaron diferencias en el peso, ya que en el grupo 1 con incrementos lentos se encuentran la mayoría de los que tienen peso $<1,000 \mathrm{~g}$ en $27.3 \%, 63.3 \%$ entre 1,000 y $1,500 \mathrm{~g}, \mathrm{y}$ el resto $>1,500 \mathrm{~g}$. A diferencia del grupo 2 donde no hubo ningún paciente $<1,000$ g, $47.1 \%$ fueron de 1,000 a 1,500 g y $52.9 \%$ con peso $>1,500 \mathrm{~g}$, y por último en el grupo 3 con incrementos rápidos fue donde hubo más pacientes con peso $>1,500 \mathrm{~g}$ con $55.6 \%$, sólo un paciente $<1,000 \mathrm{~g} \mathrm{y}$ $33.3 \%$ de 1,000 a $1,500 \mathrm{~g}$.

El grupo 1 tardó en promedio $18.4 \pm 6.66$ días en llegar a los requerimientos calóricos totales, en cambio el grupo 2 tardó $10.3 \pm 8.19$ días, y el grupo 3 con avances más rápidos tardó en promedio una semana $7.89 \pm 3.65$. Del mismo modo el uso de NPT en el grupo 1 lo requirió por casi dos semanas $13.4 \pm 7.25$, el grupo 2 por $5.32 \pm 8.49$ días y el grupo 3 menos de una semana $3.2 \pm 3.7$ días.

Se detectaron múltiples comorbilidades en su mayoría frecuentes en esta etapa de prematurez, las cuales tuvieron variación según el grupo, encontrando predominio de sepsis en 54.4\% en el grupo 1 , seguida de persistencia del conducto arterioso en
$18.2 \%$; en el grupo $2,29.4 \%$ presentó hiperbilirrubinemia multifactorial, $29.4 \%$ sin comorbilidad, con una disminución en la presencia de sepsis de $17.6 \%$, al igual que cierre de conducto arterioso; en el grupo 3 se observó $44.4 \%$ sin comorbilidad, $22.2 \%$ con hiperbilirrubinemia multifactorial, sin comorbilidades cardiacas como en los primeros dos grupos.

\section{DISCUSIÓN}

Aunque en la unidad de terapia intensiva neonatal el médico tratante decidió con qué, cómo y cuándo iniciar el estímulo enteral a los RNPT, se inició estimulación enteral dentro de las primeras 72 horas de vida. A pesar de que la fórmula más utilizada es hidrolizada, $67.5 \%$ recibió LM sola o combinada, lo cual indica que los médicos prefieren la leche humana sobre cualquier otro tipo de leche. Incrementos de $30-50 \mathrm{~mL} / \mathrm{kg} /$ día se asocian a menos días de uso de NPT y por lo tanto, a alcanzar en menos tiempo el requerimiento calórico total. Estos avances también se asociaron a menor incidencia de sepsis y de comorbilidad. No encontramos asociación en ninguno de los grupos entre ECN y aumentos rápidos, esto es de llamar la atención, ya que en la práctica se teme a esta relación.

La LM no siempre está disponible y se ha evaluado una variedad de fórmulas como fuentes nutricionales alternas para los RN PT. En nuestro estudio observamos que la mayoría recibió fórmula hidrolizada en combinación con LM y no fórmula para prematuro (sólo 10.8\%), que fueron diseñadas para esta población y tienen como característica mayor aporte calórico aproximadamente $80 \mathrm{Kcal} / 100 \mathrm{~mL}$ en comparación con la fórmula de inicio para $\mathrm{RN} 68$ $\mathrm{Kcal} / 100 \mathrm{~mL}$ y la hidrolizada $68 \mathrm{Kcal} / 100 \mathrm{~mL} .{ }^{9,12}$ Con estos datos sería interesante comparar la fórmula hidrolizada contra fórmula para prematuro dada la diferencia de calorías entre ellas y su relación con ganancia ponderal y el alcance de requerimiento calórico total.

A pesar de que los estudios que se han hecho aún no han demostrado si existe algún protocolo de alimentación óptimo, hay una marcada variación en el inicio y en los avances, si es mejor hacer incrementos rápidos (30-40 mL/kg/día) o lentos $(15-20 \mathrm{~mL} /$ $\mathrm{kg} / \mathrm{día})^{3,13}$ para lograr el crecimiento adecuado y son muchas las razones, por ejemplo, la preocupación de que los bebés prematuros son incapaces de digerir y absorber nutrientes complejos. Mucho se teme que la relación con ECN influye en la toma de decisiones. ${ }^{10,11}$ Se ha observado que cuando se detecta algo 
Tabla 3: Por grupo de incrementos.

\begin{tabular}{lccc}
\hline & $\begin{array}{c}\text { Grupo } 1(\mathrm{n}=11) \\
\mathrm{n}(\%)\end{array}$ & $\begin{array}{c}\text { Grupo 2 }(\mathrm{n}=17) \\
\mathrm{n}(\%)\end{array}$ & $\begin{array}{c}\text { Grupo } 3(\mathrm{n}=9) \\
\mathrm{n}(\%)\end{array}$ \\
$\begin{array}{l}5 \mathrm{a} 20 \mathrm{~mL} / \mathrm{kg} / \mathrm{dí} \\
30 \text { a } 50 \mathrm{~mL} / \mathrm{kg} / \mathrm{día}\end{array}$ \\
\hline $\begin{array}{l}\text { Semanas de gestación } \\
\text { Peso (gramos) }\end{array}$ & $30 \pm 1.2$ & $31.6 \pm 1.52$ & $31.3 \pm 1.6$ \\
$<1,000$ & $3(27.3)$ & $0(0.0)$ & $1(11.1)$ \\
1,000 a 1,500 & $7(63.3)$ & $8(47.1)$ & $3(33.3)$ \\
$>1,500$ & $1(9.1)$ & $9(52.9)$ & $5(55.6)$ \\
Días de vida extrauterina para llegar al & $18.4 \pm 6.66$ & $10.3 \pm 8.19$ & $7.89 \pm 3.65$ \\
requerimiento calórico total & & & $3.2 \pm 3.7$ \\
Días de uso de nutrición parenteral & $13.4 \pm 7.25$ & $5.32 \pm 8.49$ & $0(0.0)$ \\
Comorbilidades & $1(9.1)$ & $0(0.0)$ & $0(0.0)$ \\
Displasia broncopulmonar & $2(18.2)$ & $3(17.6)$ & $0(0.0)$ \\
Persistencia del conducto arterioso & $1(9.1)$ & $1(5.9)$ & $1(11.1)$ \\
Enterocolitis Grado IA & $1(9.1)$ & $0(0.0)$ & $2(22.2)$ \\
Sangrado intestinal/intolerancia vía oral & $0(0.0)$ & $5(29.4)$ & $2(22.2)$ \\
Hiperbilirubinemia multifactorial & $6(54.5)$ & $3(17.6)$ & $4(44.4)$ \\
Sepsis & $0(0.0)$ & $5(29.4)$ & \\
Ninguna & & & \\
\hline
\end{tabular}

inusual en las condiciones clínicas del paciente, los médicos inmediatamente detienen la alimentación, ya sea intravenosa y/o enteral. Este manejo reduce claramente la ingesta nutricional requerida para el crecimiento y entre las principales causas se mencionan Apgar bajo, distensión abdominal, aspirado gástrico biliar, apnea y taquipnea. ${ }^{14}$ En este estudio se observó que el grupo 1 de incrementos lentos tiene como característica que más de $50 \%$ tuvieron sepsis durante su internamiento y éste podría ser un factor determinante en la decisión de los médicos entre un inicio tardío de vía oral o incrementos lentos; sin embargo, también describimos que en nuestra población no hay asociación con ECN en ninguno de los grupos.

Una de las limitantes de nuestro estudio es el número de pacientes en contraste con la diversidad en el manejo de cada médico tratante, ya que hubo quienes iniciaban tardíamente la vía enteral sin un criterio específico y por lo tanto, pudimos comparar muchos tipos de incrementos.

\section{CONCLUSIÓN}

Los incrementos rápidos en la vía enteral de 30-50 $\mathrm{mL} / \mathrm{kg} /$ día de los RN PT reducen el tiempo en llegar a los requerimientos calóricos totales y el uso de NPT. Esta práctica no incrementa el riesgo de ECN, por lo contrario está asociada a menos comorbilidades. Se requieren estudios en el futuro para determinar el efecto a largo plazo especialmente con prematuros moderados y extremos.

\section{REFERENCIAS}

1. Kennedy K, Tyson J. Rapid versus slow rate of advancement of feedings for promoting growth and preventing necrotizing enterocolitis in parenterally fed low-birth-weight infants. Cochrane Database Syst Rev. 1998; (4): 1-11.

2. Bozzetti V, Tagliabue P. Enteral nutrition for preterm infants: by bolus or continuous? An update. Pediatr Med Chir. 2017; 39 (2): 67-70.

3. Mena P. Cuándo y cómo iniciar la alimentación enteral en el prematuro extremo. Rev Chil Pediatr. 2001; 72 (3): 247-250.

4. Viswanathan S, Merheb R, Wen X, Collin M, Groh-Wargo S. Standardized slow enteral feeding protocol reduces necrotizing enterocolitis in micropremies. J Neonatal Perinatal Med. 2017; 10 (2): 171-180.

5. Karagol B, Zenciroglu A, Okumus N, Polin R. Randomized controlled trial of slow vs rapid enteral feeding advancements on the clinical outcomes of preterm infants with birth weight 750-1,250 g. J Parenter Enteral Nutr. 2012; 37 (2): 223-228.

6. Hans D, Pylipow M. Nutritional practices in the neonatal intensive care unit: analysis of a 2006 neonatal nutrition survey. Pediatrics. 2009; 123(1):51-57.

7. Modi M, Ramji S. Early aggressive enteral feeding in neonates weighing 750-1,250 grams: a randomized controlled trial. Indian Pediatr. 2019; 56 (4): 294-298.

8. Ben XM. Nutritional management of newborn infants: practical guidelines. World J Gastroenterol. 2008; 14 (40): 6133-6139.

9. Henderson G, Anthony M, McGuire W. Formula milk versus preterm human milk for feeding preterm or low birth weight infants. Cochrane Database Syst Rev. 2001; (3): CD002972. 
An Med (Mex) 2021; 66 (1): 13-18

10. Oddie S, Young L, McGuire W. Slow advancement of enteral feed volumes to prevent necrotising enterocolitis in very low birth weight infants. Cochrane Database Syst Rev. 2017; 8 (8): 1-43.

11. Dorling J, Abbott J, Berrington J, Bosiak B, Bowler U, Boyle E et al. Controlled trial of two incremental milk-feeding rates in preterm infants. N Engl J Med. 2019; 381 (15): 1434-1443.

12. Udaeta-Mora E, Toussaint MG, Pérez-Marín P, CarmonaRodríguez M. Alimentación enteral en el recién nacido pretérmino y de término con bajo peso: estado actual en México. Gaceta Med Mex. 2005; 141 (4): 283-290.

13. Alyahya W, Simpson J, Garcia AL, Mactier H, Edwards CA. Early versus delayed fortification of human milk in preterm infants: a systematic review. Neonatology. 2020; 117 (1): 24-32.

14. Hay W. Aggressive nutrition of the preterm infant. Curr Pediatr Rep. 2013; 1 (4): 229-239. 